\title{
Percutaneous Coronary Intervention of Anomalous Right Coronary Artery Arising from the Left Coronary Cusp using an Undersized Judkins Catheter through Transradial Route
}

Ram Awatar Rawat*, Dinesh Choudhary, Rakesh Mahla, Sunil K Budania and Pintoo Nahata

Department of Cardiology, HRMC Cardiac Center, PBM Hospital, S P Medical College, Bikaner, India

\begin{abstract}
Coronaries with anomalous origin are difficult to visualize on coronary angiography. Percutaneous coronary intervention $(\mathrm{PCl})$ is a difficult procedure in these vessels because of difficulty in cannulation and poor guide support. The case of a 54-year-old gentleman who presented with an acute inferior wall myocardial infarction from occlusion of an anomalous right coronary artery arising from the left coronary cusp is described.
\end{abstract}

Keywords: Cannulation; Coronary; Judkins catheter

\section{Case Report}

Prevalence of anomalous coronary arteries is $1 \%$ during elective coronary angiography. An anomalous origin of the Right Coronary Artery (RCA) from the left coronary cusp is even rare $(8 \%-16 \%$ of all coronary anomalies) [1], usually from the orifice anterior to the left main coronary ostium and courses inter-arterially [1].The anomalous origin of RCA from the left coronary sinus is found in some necropsy studies of young individuals with sudden, unexplained death [2]. The risk is not as much as with an anomalous left coronary artery from the right sinus with an inter-arterial course. This anomaly may coexist with atherosclerotic disease. Percutaneous Coronary Intervention (PCI) of anomalous RCA is challenging because of cannulation difficulties and suboptimal guide catheter support due to anterior and noncircular orifice. We are reporting a case of an acute ST-segment elevated inferior wall myocardial infarction (thrombolysed with streptokinase elsewhere) caused by atherosclerotic disease of an anomalous right coronary artery from the left coronary cusp. Coronary angiogram was performed through the right radial route with Tiger 3.5 catheter $(5 \mathrm{Fr})$, revealing insignificant disease in left system. Cannulation of the RCA was achieved with difficulty by same catheter and revealed a $90 \%$ lesion in the mid-portion of the right coronary artery. Subsequently, PCI was performed with use of a 6 Fr Judkins left $3.0 \mathrm{~cm}$ guiding catheter (Figures 1-3), which provided excellent support through right radial route.

\section{Discussion}

PCI of anomalous right coronary artery is challenging because

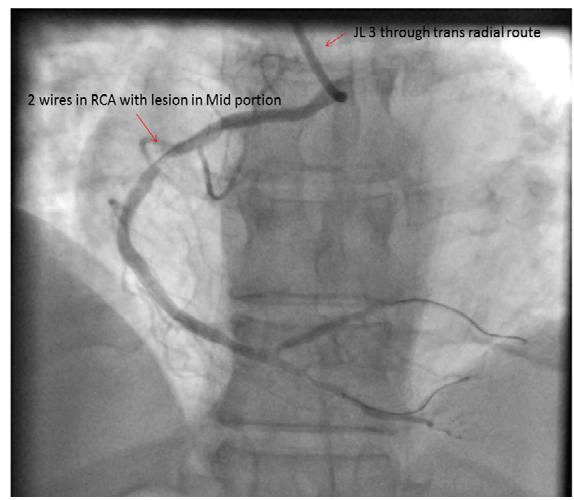

Figure 1: $\mathrm{PCl}$ of RCA: two wires parked in RCA distally with $\mathrm{JL} 3$ guiding catheter support through trans-radial route. of unusual location of the ostium [3] and suboptimal guide catheter support, for which several strategies have been described [4]. Charney $\mathrm{R}$ et al. [4] and Yip $\mathrm{H}$ et al. [5] used Amplatz left guiding catheter, while Praharaj TK used Voda guiding catheter [6] for cannulation of anomalous RCA (ARCA ). Use of oversized $[7,8]$ as well as undersized Judkins left guide catheters for this anomaly through femoral route has been well described. We used undersized Judkinleft catheter through trans-radial route and found this approach to be particularly useful The undersized Judkins catheter tip could be directed easily in a coaxial manner to the ostium of the anomalous right coronary artery which provided good support for the procedure. The purpose of the case report is to show that anomalous RCA angioplasty can be done easily

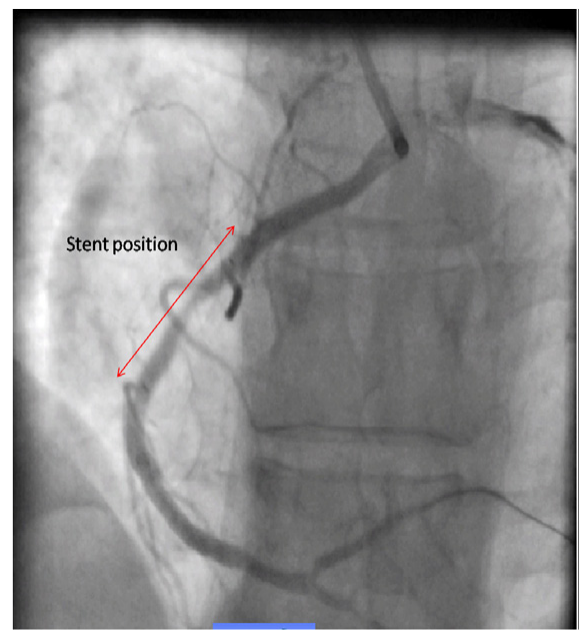

Figure 2: Stent implantation: Anomalous RCA hooked with JL 3 guiding catheter through trans-radial route.

*Corresponding author: Ram Awatar Rawat, Department of Cardiology, HRMC Cardiac Centre, PBM Hospital, S.P. Medical College, Bikaner, India, Tel: +91 151 222 6300; E-mail: ramdmcardiology@gmail.com

Received September 01, 2014; Accepted September 29, 2014; Published October 01, 2014

Citation: Rawat RA, Choudhary D, Mahla R, Budania SK, Nahata P (2014) Percutaneous Coronary Intervention of Anomalous Right Coronary Artery Arising from the Left Coronary Cusp using an Undersized Judkins Catheter through Transradial Route. J Clin Case Rep 4: 440. doi:10.4172/2165-7920.1000440

Copyright: (c) 2014 Rawat RA, et al. This is an open-access article distributed under the terms of the Creative Commons Attribution License, which permits unrestricted use, distribution, and reproduction in any medium, provided the original author and source are credited. 
Citation: Rawat RA, Choudhary D, Mahla R, Budania SK, Nahata P (2014) Percutaneous Coronary Intervention of Anomalous Right Coronary Artery Arising from the Left Coronary Cusp using an Undersized Judkins Catheter through Transradial Route. J Clin Case Rep 4: 440. doi:10.4172/2165-7920.1000440

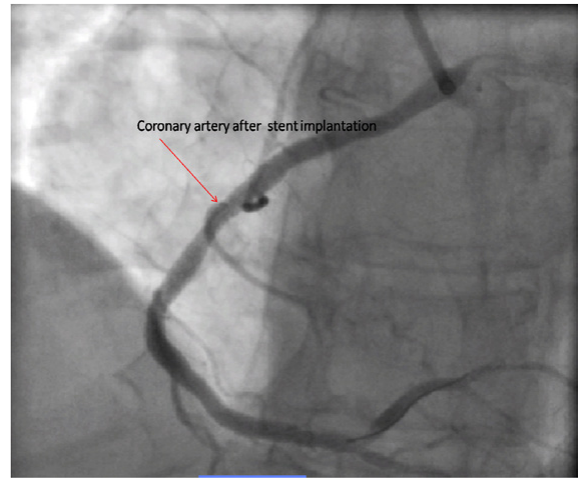

Figure 3: After stent implantation in RCA

through trans-radial route as this route is having distinct advantages over trans-femoral route [9]. Right radial approach has been described to be successful in accessing anomalous left sinus of valsalva origin right coronary artery [10]. By traversing a shorter segment of aorta, using trans radial route, the effects of the distal anatomy on proximal catheter behavior are decreased. This is especially noticeable in right coronary interventions. The right coronary catheters when placed from right radial access, provide superior back-up support, as opposed to the support provided by the same catheter from femoral approach, in view of the fact that transradial placement allows for these catheters to obtain added support from the contralateral aortic wall, similar to the behavior of the extra-backup series guide catheters for the left coronary artery.

\section{References}

1. Yamanaka O, Hobbs RE (1990) Coronary artery anomalies in 126,595 patients undergoing coronary arteriography. Cathet Cardiovasc Diagn 21: 28-40.

2. Roberts WC, Siegel RJ, Zipes DP (1982) Origin of the right coronary artery from the left sinus of valsalva and its functional consequences: analysis of 10 necropsy patients. Am J Cardiol 49: 863-868.

3. Jim MH, Siu CW, Ho HH, Miu R, Lam YM, et al. (2005) Anomalous origin of right coronary artery from the left coronary sinus: incidence, characteristics, and a systematic approach for rapid diagnosis. J Interv Cardiol 18: 101-106.

4. Charney R, Spindola-Franco H, Grose R (1993) Coronary angioplasty of anomalous right coronary arteries. Cathet Cardiovasc Diagn 29: 233-235.

5. Yip H, Chen MC, Wu CJ (2001) Primary angioplasty in acute inferior myocardial infarction with anomalous-origin right coronaryarteries as infarct-related arteries: Focus on anatomic and clinical features, outcomes, selection of guiding catheters and management. J Invasive Cardiol 13: 290-297

6. Praharaj TK, Ray G (2001) Percutaneous transluminal coronary angioplasty with stenting of anomalous right coronary artery originating from left sinus of Valsalva using the Voda guiding catheter: a report of two cases. Indian Heart J 53: 79-82.

7. Cohen MG, Tolleson TR, Peter RH, Harrison JK, Sketch MH (2002) Successfu percutaneous coronary intervention with stent implantation in anomalous right coronary arteries arising from theleft sinus of valsalva: A report of two cases. Catheter Cardiovasc Interv 55: 105-108.

8. Ng W, Chow WH (2000) Successful angioplasty and stenting of anomalous right coronary artery using a 6 French Left Judkins \#5 guide catheter. J Invasive Cardiol 12: 373-375

9. Rao SV, Ou FS, Wang TY, Roe MT, Brindis R, et al. (2008) Trends in the prevalence and outcomes of radial and femoral approaches to percutaneous coronary intervention: a report from the National Cardiovascular Data Registry. JACC Cardiovasc Interv 1: 379-386.

10. Lorin JD, Robin B, Lochow P, Lorenzo A, Sedlis SP (2000) The right radia approach for stenting of lesions in the right coronary artery with anomalous take-off from the left sinus of valsalva. J Invasive Cardiol 12: 478-480. 